\title{
Type III Interferons: Emerging Roles in Autoimmunity
}

\author{
Sindhu Manivasagam ${ }^{1,2}$ and Robyn S. Klein ${ }^{1,2,3,4^{*}}$ \\ ${ }^{1}$ Center for Neuroimmunology \& Neuroinfectious Diseases, Washington University School of Medicine, St. Louis, \\ MO, United States, ${ }^{2}$ Department of Medicine, Washington University School of Medicine, St. Louis, MO, United States, \\ ${ }^{3}$ Department of Pathology \& Immunology, Washington University School of Medicine, St. Louis, MO, United States, \\ ${ }^{4}$ Department of Neurosciences, Washington University School of Medicine, St. Louis, MO, United States
}

\section{OPEN ACCESS}

Edited by: Adriana Forero,

The Ohio State University, United States

Reviewed by:

Noa B. Martin-Cofreces, Princess University Hospital, Spain Arantzazu Alfranca,

Hospital de la Princesa, Spain

*Correspondence:

Robyn S. Klein rklein@wustl.edu

Specialty section:

This article was submitted to Cytokines and Soluble Mediators in Immunity, a section of the journal

Frontiers in Immunology

Received: 24 August 2021

Accepted: 11 October 2021

Published: 26 November 2021

Citation:

Manivasagam $S$ and Klein RS (2021) Type III Interferons: Emerging Roles in Autoimmunity.

Front. Immunol. 12:764062. doi: 10.3389/fimmu.2021.764062
Type III interferons (IFNs) or the lambda IFNs (IFNLs or IFN- $\lambda s$ ) are antimicrobial cytokines that play key roles in immune host defense at endothelial and epithelial barriers. IFNLs signal via their heterodimeric receptor, comprised of two subunits, IFNLR1 and interleukin (IL)10R $\beta$, which defines the cellular specificity of the responses to the cytokines. Recent studies show that IFNL signaling regulates CD4+ T cell differentiation, favoring Th1 cells, which has led to the identification of IFNL as a putative therapeutic target for autoimmune diseases. Here, we summarize the IFNL signaling pathways during antimicrobial immunity, IFNL-mediated immunomodulation of both innate and adaptive immune cells, and induction of autoimmunity.

Keywords: interferon lambda, autoimmune diseases, Th1 cells, IFNLR, neuroimmunology and neuropathology

\section{INTRODUCTION}

Type III interferons (IFNs) or the lambda IFNs (IFNLs or IFN- $\lambda s$ ), generate and sustain antiviral and immunomodulatory cellular responses. Specifically, they are known for their ability to control viral replication and infection at barrier surfaces, such as the epithelium of the lung and gut, blood brain barrier, and placenta (1), and at the liver (2). Type III interferons consist of three different functional genes in humans, IFNL1, IFNL2, IFNL3, and one pseudogene IFNL4 (3-5). Mice have two functional genes, Ifnl2 and Ifnl3, and pseudogene Ifnl1. Type III interferons are closely related to type I IFN, signaling through common Janus Kinase and Signal Transducer and Activator of Transcription (JAK-STAT) pathways that lead to transcription of IFN-stimulated genes (ISGs) (1, 6, 7). Specifically, type I IFN binds to the IFN $\alpha \beta$ receptor (IFNAR) and type III IFN binds to the heterodimeric receptor (IFNLR), which is comprised of two subunits, IFNLR1 and interleukin (IL) $10 R \beta(3,4)$. Binding of these ligands to their receptors leads to downstream phosphorylation of Signal Transducer and Activator of Transcription (STAT) 1 and STAT2, subsequent recruitment of interferon regulatory factor (IRF)9, and transcription of interferon-stimulated genes (ISGs) $(3,8)$. Unlike type I IFN, however, type III IFN do not upregulate IRF1 that leads to downstream production of pro-inflammatory cytokines (9).

Despite similarities in downstream signaling between type I and type III IFN, they differ in cellular expression of their receptors. Since IFNAR is ubiquitously expressed, type I IFN-inhibition of viral replication occurs in many cell types. In contrast, IFNLR-mediated antiviral responses exhibit specificity for viruses that replicate at barrier surfaces due to its cell-specific expression $(1,10)$. In both mice and humans, IFNLR is expressed by epithelial cells (10), endothelial cells of the blood-brain barrier (11), macrophages (12-15), subsets of DCs (16-20), and neutrophils (21, 22). In humans, B cells have also 
been shown to respond to IFNL (23), while data regarding T cell responsiveness is inconclusive. The limited range of expression of IFNLR1 to mucosal surfaces and specific immune cells offers large potential for type III IFN to be used as therapeutic targets, given their higher tissue specificity and lower likelihood for off target effects, when compared to type I IFN.

In addition to differences in receptor expression, type I and type III IFN have different kinetics. Following hepatitis C infection of cultured cells, the type I IFN response is rapidly induced, while the type III IFN is slower and remains sustained for a longer duration $(24,25)$; similar kinetic differences are observed following aspergillus fumigatus infection in mice as well (22). Furthermore, treatment of human hepatocytes with type III IFN leads to a delayed and slow induction of ISGs, such as ISG15, interferon-induced GTP-binding protein $M X 1$, and 2'5 '-oligoadenylate synthetase (OAS1), compared to type I IFN which leads to a faster and transient induction (9). These kinetic differences suggest that type I IFN may play a significant role early during acute infection, while type III IFN may promote long term control. Overall, the differences in cellular targets, kinetics of transcriptional effects, and immunomodulatory effects distinguish the effects of type I and III IFNs, which have led to studies examining type III IFN effects on immune cells in noninfectious contexts, including cancers and autoimmune diseases. Here we review the effects of type III IFN on leukocytes, certain cancers, and autoimmune diseases.

\section{IMMUNE MODULATION}

\section{T Cell-Dendritic Cells Axis for IFNL Responses}

Many studies have suggested an important role for type III IFN in $\mathrm{T}$ cell polarization, with most providing evidence that IFNL down-regulates Th2 polarization and sustains Th1 activation (17, 26-30) (Figure 1). Intranasal, therapeutic administration of IFNL2/3 in a murine model of Th2-driven allergic asthma mitigated lung pathology and diminished levels of epithelial secreted Th2 cytokines, thymic stromal lymphopoietin (TSLP) and IL-33 in the bronchoalveolar lavage fluid (26). Additionally, adenovirus mediated expression of human IFNL1 in a murine asthma model led to attenuated eosinophilia, diminished antigen specific Th2 responses, and promotion of regulatory $\mathrm{T}$ (Treg) responses (31). IFNLs may also influence Th17 polarization, as treatment with IFNL2 reduced numbers of Th17 and $\gamma \delta \mathrm{IL}-17+$ cells in the inflamed joint compared to vehicle treatment in a model of collagen induced arthritis (32) and suppressed T cell IL17 secretion in mediastinal lymph nodes of mice with allergic airway disease compared with vehicle treatment (17).

Studies of IFNL-mediated modulation of human immune cells have demonstrated similar Th1 promoting phenotypes. In vitro treatment of human peripheral blood mononuclear cells (PBMCs) with increasing concentration of IFNL1 increased production of the Th1 cytokine IFN $\gamma$ and decreased production of Th2 cytokines interleukin (IL)-13, IL-4, and IL-5, with IL-13 expression being most consistently diminished following IFNL1 treatment $(27,28,33)$.
Treatment of human breast tumor suspensions with IFNL1 also induced expression of IL-12p40, a key driver of Th1 polarization (34). Effects of IFNLs on T cells may also be temporally dependent. In a model of murine lymphocytic choriomeningitis virus (LCMV) infection, IFNL dampened acute $\mathrm{T}$ cell responses, but promoted chronic $\mathrm{T}$ cell responses (35).

Given that IFNL has robust effects on $\mathrm{T}$ cell responses, it raises the question of whether IFNL acts directly or indirectly on $\mathrm{T}$ cells. Studies suggest that $\mathrm{T}$ cells do not respond to IFNL stimulation. T cells express low levels of Ifnrl1 mRNA (21). In vitro treatment of T cells with IFNL failed to induce downstream phosphorylation of STAT1 or STAT3 (36) or expression of ISGs, such as CXCL10, ISG15, and protein kinase R (EIF2AK2) (20). Data instead suggest that IFNL may act on DCs to modulate and augment downstream $\mathrm{T}$ cell polarization and effector function $(17,18)$. IFNL2 treatment of lung and bone marrow derived CD11 ${ }^{+}$DCs increased expression of both Tbet and $I L-12$ (17). Furthermore, co-culture of IFNL2 treated DCs and ovalbumin (OVA) peptide-specific $\mathrm{CD} 4^{+} \mathrm{T}$ cells led to increased production of IFN $\gamma$ and diminished production of IL-5, IL-13, and IL-17 (17). Transfer of these IFNL2 treated DCs to animals prior to OVA challenge skewed peripheral lymph node immune responses towards a Th1 phenotype and subsequently suppressed allergic airway disease (17). In a murine model of influenza A viral (IAV) infection Ifnlr1 mRNA expression on DCs increased following infection, which correlated with increased IFN $\gamma$ and tumor necrosis factor (TNF) production by lung $\mathrm{CD}^{+} \mathrm{T}$ cells. Conditional deletion of Ifnlr 1 on CD $11 \mathrm{c}^{+} \mathrm{DCs}$ in this model decreased numbers of infiltrating $\mathrm{CD}^{+} \mathrm{T}$ cells in the lungs (18). Comparison of wild-type (WT) and Ifnlr $1^{-/-}$DCs also showed functional differences, as $I f n l r 1^{-/-}$DCs showed diminished CD40 expression compared to WT DCs and ability to uptake and process antigen (18). Type III interferon mediated modulation of human DCs shows both similarities and differences compared to murine DCs. In vitro treatment of human peripheral DCs with IFNL increased MHC class I and class II expression, but did not have strong effects on CD80 or CD40 (19). Analysis of mixed lymphocyte reactions of human monocyte derived DCs (mDCs) with naïve T cells under various T cell polarizing conditions led to reduced IL-13 expression upon addition of IFNL (28). Another study of IFNL treated DCs cocultured with $\mathrm{CD} 4^{+} \mathrm{T}$ cells showed increased proliferation of $\mathrm{CD} 4{ }^{+} \mathrm{CD} 25^{+} \mathrm{FoxP}^{+}$Treg cells (37). Together, these data suggest that DCs are key mediators of IFNL driven T cell responses.

In addition to responding to IFNL, conventional DCs (cDC) can also produce IFNL (34). Following administration of polyinosinic:polycytidylic acid (polyI:C), murine $\mathrm{CD} \mathrm{a}^{+} \mathrm{cDCs}$ and human $\mathrm{CD}_{141^{+}}\left(\mathrm{BDCA}^{+}\right)$DCs produce significant quantities of IFNL1; in mice this depended on activation of toll-like receptors (TLR)3, IRF3, IRF7, and IRF8 (38). In corroboration, co-culture of a hepatitis $\mathrm{C}$ infected hepatoma cell line with human PBMCs increased IFNL production by $\mathrm{CD}_{141}{ }^{+}\left(\mathrm{BDCA}^{+}\right)$DCs (39). In a non-viral model, Hubert et al. demonstrated that human breast tumor associated cDCs upregulate IFNL1 gene and protein expression compared to adjacent normal tissue (34). Similar to cDCs, human 


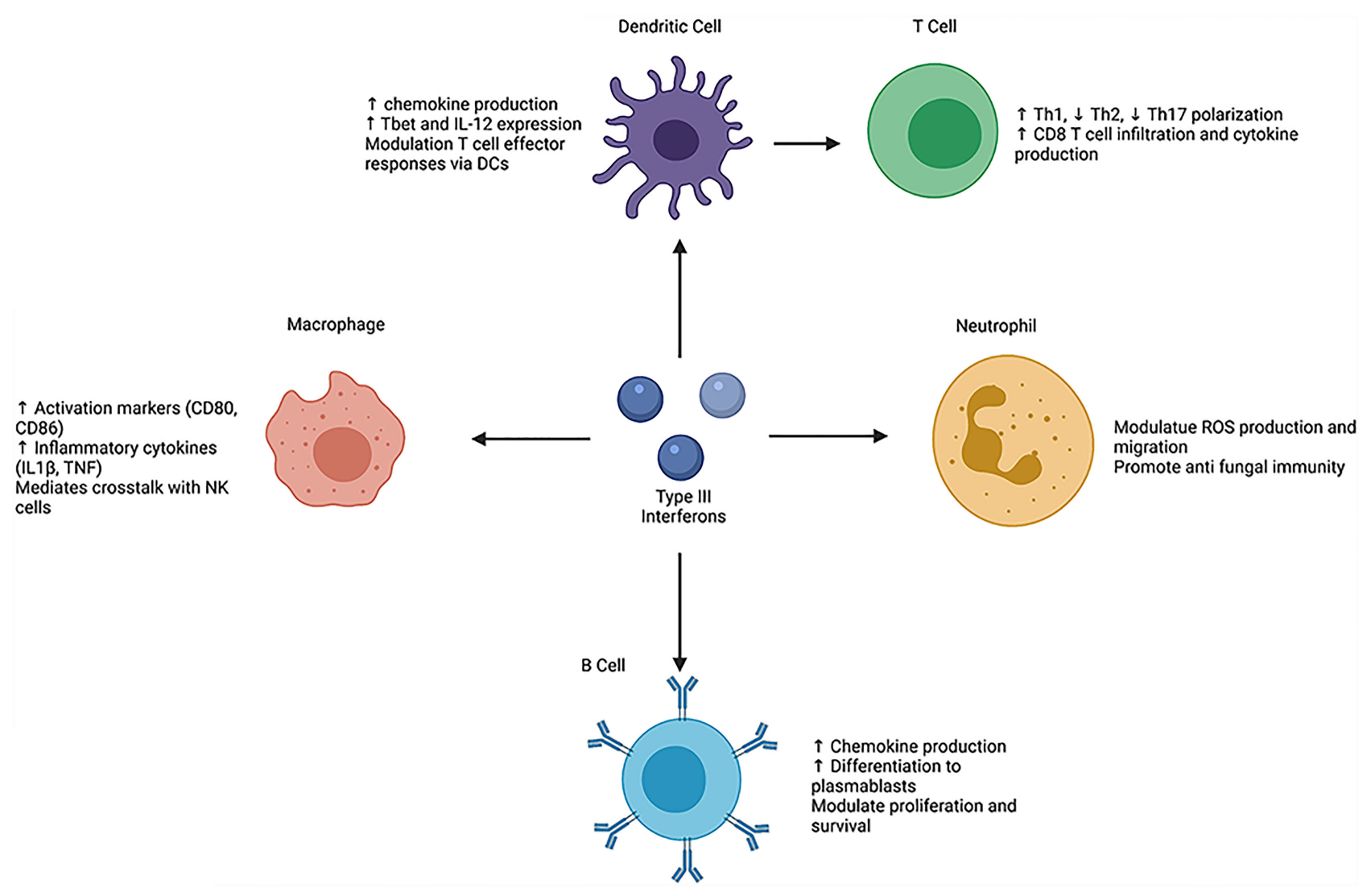

FIGURE 1 | Schematic overview of the direct and indirect effects of type III interferons on leukocyte phenotypes and functions. helper T cell (Th), T-box transcription factor (Tbet), interleukin (IL), tumor necrosis factor (TNF), natural killer (NK), reactive oxygen species (ROS). Created with BioRender.com.

plasmacytoid DCs (pDCs) can both produce and respond to IFNL. pDCs produce IFNL in response to herpes simplex virus, influenza virus, sendai virus, and HIV-1. Treatment of PBMCs with IFNL led specifically to upregulation of MHC class I and CD83 on pDCs but not other immune cell types, suggesting IFNL can act in an autocrine manner (16).

Aside from modifying DC mediated T cell activation and costimulation, IFNL may modulate chemokine driven migration and tissue specific infiltration of $\mathrm{T}$ cells. It has been established that type II IFN (IFN $\gamma$ ) induces downstream release of CXCL9, CXCL10, and CXCL11 (40, 41), chemoattractants responsible for guiding lymphocyte migration. Type III IFN treatment of human peripheral blood mononuclear cells also elevated expression of CXCL9, CXCL10, and CXCL11, independently of type II IFN signaling (42). CXCL10, in particular, is a key chemokine that recruits $\mathrm{T}$ cells to sites of inflammation and maintains their Th1 cell effector function, as the CXCL10 receptor, CXCR3, is preferentially expressed on Th1 cells compared to Th2 cells $(40,43,44)$. These data suggest one mechanism by which IFNL treatment of cells promotes Th1 cell recruitment and maintenance. Recently, Goel et al. confirmed that type III IFN can upregulate CXCL10, as treatment of an immortalized human keratinocyte cell line with IFNL1 for 24 hours increased CXCL10 production compared with both vehicle and IFN $\alpha$ treatments. Additionally, in this model,
IFNL1 treatment did not significantly increase CXCL9 levels compared to vehicle, and both IFNL1 and IFN $\alpha$ increased CXCL11 levels to a similar extent (45). In human breast tumors, Hubert et al. showed that cytokine analysis of the soluble tumor milieu demonstrated correlations between IFNL1 expression and CXCL10, CXLC11, and CXCL9, in addition to TNF and IL-12p40. Treatment of human breast tumor suspensions with IFNL1 resulted in increased expression of CXCL10 and CXCL11 (34). In contrast, treatment of immortalized human hepatocytes with IFNL3 failed to induce CXCL10 gene or protein expression (9). These data suggest that IFNL does not induce the same chemokine response in all cells, and instead promotes cell-specific chemokine responses. These differences may arise from the activation of non-canonical signaling pathways in certain cell types.

\section{Macrophage - NK Cell Axis}

IFNL also alters human macrophage phenotypes, as fully differentiated macrophages derived from monocytes gain expression and functional capacity of the IFNLR1 receptor (12, 15) (Figure 1). In vitro studies show that macrophage treatment with all IFNLs inhibits replication of human immunodeficiency virus type I (HIV-1) via induction of JAK-STAT signaling (13, 46). Following IFNL3 treatment, macrophages upregulate ISG15, immune cell activation proteins, such as CD80 and CD86, and 
inflammatory cytokine production such as IL1 $\beta$ and TNF (12, 14). When treated with IFNL1 in the presence of a TLR7/8 agonist or lipopolysaccharide (LPS), human monocyte derived macrophages also increase production of IL-12p40; these effects are not observed upon treatment with IFN $\alpha$ and are thus specific to IFNL $(14,15)$. This increased IL-12 drives IFN $\gamma$ production by natural killer (NK) cells. Incubation of NK cells with supernatants from macrophages treated with IFNL, LPS, and IFN $\gamma$ increased $\mathrm{NK}$ cell IFN $\gamma$ production compared with IFN $\alpha$, LPS and IFN $\gamma$ treatment (15). Wang et al. showed further evidence of IFNL-mediated macrophage NK cell crosstalk. During murine influenza A (IAV) infection, IFNL exerted antiviral activity by increasing NK cell numbers and promoting NK maturation. However, depletion of macrophages in this model reversed the effects of IFNL on NK cells (47). Beyond these studies, the ability of IFNL to promote NK cellmacrophages interaction is not well understood and requires further investigation in autoimmune diseases and cancer.

\section{B Lymphocyte Responses to IFNL}

Human naïve and memory B cells express IFNLR1 $(20,48)$ and induce STAT1 phosphorylation following treatment with IFNL, suggesting that B cells are functionally responsive to IFNL (23) (Figure 1). B cell signaling occurs through the JAK-STAT pathway, as treatment with a JAK inhibitor prevented phosphorylation of STAT1 (23). B cell stimulation with IFNL and TLR7/8 agonist led to upregulation of only CD69, and no change in other co-stimulatory molecules, such as CD40, CD80, and CD86 (48). IFNL treatment of B cells also upregulated production of chemokines such as CXCL9, CXCL10, and CXCL11. Addition of IFNL following B cell receptor (BCR) stimulation upregulated the mechanistic target of rapamycin complex 1 (mTORC1) signaling pathway compared to BCR stimulation alone and promoted transcription of genes involved in differentiation of naïve B cells to plasmablasts (23). IFNL3 pretreatment of B cells (derived from healthy individuals) prior to stimulation with $\mathrm{H} 1 \mathrm{~N} 1$ reduced their proliferative capacity and IgG production (29). Novak et al. showed that a multiple myeloma cell line was responsive to IFNL, as IFNL1 treatment increased cell proliferation and promoted cell survival (49). In contrast to human B cells, murine B cells express low levels of Ifnlr1 mRNA as measured by quantitative PCR and do not upregulate downstream ISGs in response to IFNL (21).

\section{Functional Regulation of Neutrophils by IFNL}

IFNL binds to IFNLR on both murine and human neutrophils to alter their function (Figure 1). In in vitro studies, IFNL treatment of bone-marrow-derived neutrophils following stimulation with LPS or TNF resulted in diminished release of reactive oxygen species (ROS) and impaired degranulation (21). In vivo administration of IFNL diminished neutrophil secretion of IL$1 \beta$ (32). IFNL also limits neutrophil migration, as IFNL2 treatment diminished neutrophil infiltration into the site of inflammation in a model of collagen induced arthritis (32). In a murine model of Aspergillus fumigatus infection, neutrophils upregulate IFNLR1 expression and type III IFNs promote their ROS generation (22).

\section{AUTOIMMUNE DISEASES}

Recent studies highlight that IFNL plays a significant role in immune-driven diseases; these diseases will be discussed in detail in the subsequent sections.

\section{Systemic and Cutaneous Lupus Erythematosus}

Systemic lupus erythematosus (SLE) is an autoimmune and inflammatory disease that affects a number of organ systems including the skin, kidneys, and brain (50). In SLE there is increased activation of $B$ cells by B cell activating factor (BAFF), TNFo, IL-6, and IL-21, in addition to increased production of autoantibodies by plasma cells. For this reason, initial SLE therapeutics, such as belimumab (anti BAFF), were targeted towards B cells $(51,52)$. Recently, other therapeutic targets have also been explored. For example, a recent phase 3 clinical trial demonstrated efficacy of anifrolumab, which targets the type I IFN receptor, in reducing SLE severity (53). The role of IFNL in SLE has been recently discussed in detail (54), and will be reviewed briefly here. Wu et al. showed that SLE patients had increased serum levels of IFNL compared to healthy controls and those with active disease had higher serum levels of IFNL compared to those with inactive disease (55). On the other hand, a study by Lin et al. did not observe a significant difference in IFNL levels between SLE patients and healthy controls. This discrepancy could be due to differences in limits of detection between the two studies. The study by Lin et al. had a larger percentage of serum values that fell below the limit of detection in healthy controls compared to SLE patients (56). Further analysis showed that SLE patients with renal or arthritis disease complications had increased serum IFNL levels compared to those without involvement of those organs $(55,57)$. In corroboration, single nucleotide polymorphisms (SNPs) at the IFNL gene locus have been correlated with risk of lupus nephritis $(58,59)$. Patients with active cutaneous lupus erythematosus (CLE) also have increased IFNL levels in their serum and skin lesions compared with healthy controls. Poly : IC exposure of epidermal explants from patients stimulated skin keratinocytes to further produce IFNL (60). Murine studies further highlight the ability of IFNL to promote SLE pathogenesis and suggest potential mechanisms. In a murine model of TLR7-induced lupus, IFNL promoted immune dysregulation through expansion of myeloid and $\mathrm{T}$ cell populations in the spleen and blood and induction of chemokine production by keratinocytes. Levels of serum IFNL and pDC derived IFNL were increased in mice following disease induction. Loss of IFNLR alleviated lupus induced splenomegaly and decreased numbers of splenic neutrophils, DCs, monocytes, $\mathrm{CD}^{+} \mathrm{T}$ cells and $\mathrm{CD} 8^{+} \mathrm{T}$ cells compared to WT animals. Analysis of murine skin revealed diminished inflammation, as $I f n l r 1^{-1-}$ animals had fewer infiltrating $\mathrm{T}$ cells, $\mathrm{B}$ cells, macrophages and neutrophils compared to WT animals. Lupus induced renal pathology was also significantly alleviated in $I f n l r 1^{-/-}$mice 
compared to WT animals (45). Together, these data suggest that type III interferons may promote autoimmune disease and pathology in the contexts of systemic and cutaneous lupus erythematosus.

\section{Arthritis}

In rheumatoid arthritis (RA) overactivation of multiple inflammatory pathways leads to subsequent inflammation of the synovium and cellular damage within joints (61). IFNL protein levels are upregulated in the sera of patients with rheumatoid arthritis compared with healthy controls (62-65); more specifically IFNL1 and IFNL2 levels are upregulated in patients with active disease (64). Disease severity in RA patients can be correlated with the presence of anti-cyclic citrullinated peptide (anti-CCP) antibodies (66). Analysis of IFNL levels in patients with and without anti-CCP antibodies showed increased IFNL1 in RA patients with detectable anti-CCP antibodies compared to RA patients negative for anti-CCP antibodies and healthy controls (65). This suggests a correlation between IFNL and disease activity in anti CCP antibody positive RA patients (62). Real time PCR analysis revealed increased expression of the receptor, IFNLR1, on PBMCs in RA patients compared with healthy controls (62). Treatment of a RA fibroblast cell line with IFNL1 upregulated cytokines IL-6, IL-8, and MMP-3 and downregulated the cytokine IL-10 (62). IFNL may also contribute to inflammation and cartilage degradation during osteoarthritis (OA) (67). OA patients had significantly increased serum IFNL1 levels and PBMC expression of IFNLR1 compared to healthy controls (67). Similarly to RA, treatment of OA fibroblasts with IFNL1 induced expression of IL-1 $\beta$, IL-6, IL-8, and MMP-3. In contrast, in a mouse model of rheumatoid arthritis, treatment with IFNL2 prevented arthritis progression, resolved inflammation, and improved pathology in comparison to a vehicle treated group (32). These data suggest isorform-specific IFNL may differentially impact inflammatory processes that mediate the pathogenesis of both OA and RA.

\section{Allergic Airway Disease}

Allergic airway diseases are characterized by chronic Th2 driven inflammation of the upper or lower airways, eosinophilic accumulation, and IgE production (68). Studies of allergic airway disease in mice reveal the ability of IFNL to inhibit Th2 polarization (26) and promote IFN $\gamma$ production (17), indicating that IFNL may enhance Th1 function (69). As discussed previously, therapeutic treatment of mice with IFNL following induction of murine asthma resulted in improved lung pathology and decreased production of Th2 cytokines such as TSLP and IL33 (26). Furthermore, Ifnlr $1^{-/-}$animals had exacerbated allergic airway disease, increased Th2 responses and IgE levels compared to WT animals (17).

Studies in humans have shown variable correlations between type III interferons and asthma. At baseline, both children and adults with asthma have increased levels of IFNL2 in their sputum compared with healthy controls; asthmatic children also had increased levels of IFNL1 in their sputum (70). Another study demonstrated that adults with asthma had increased levels of IFNL1 compared with healthy controls and that these elevated
IFNL1 levels correlated with presence of neutrophilia in the sputum (71). In contrast, primary bronchial epithelial cells and bronchoalveolar lavage cells isolated from asthmatic patients demonstrated diminished production of IFNL2/3 following infection with rhinovirus in comparison with cells from healthy control patients. The decreased production of IFNL2/3 correlated with increased viral loads in the epithelial cells isolated from asthmatic patients compared to healthy controls (72). Since asthma exacerbations are frequently instigated by viral infections (73), it is important to further clarify the role IFNs play in the context of anti-viral immunity and airway inflammation.

\section{Inflammation of the Gastrointestinal System}

Patients with inflammatory bowel disease (IBD) and mice with colitis demonstrate increased levels of IFNL and IFNLR1 compared with controls (74). Specifically, lamina propria and intestinal epithelial cells (IECs) in colon tissues of patients with IBD, and mice with colitis, exhibit increased expression of IL28 and IL28R, respectively, compared with controls. In addition, in vitro studies demonstrated that IL28 induces phosphorylation (activation) of STAT1 in IECs, leading to their proliferation in organoid culture. In a murine model of immune activation induced colitis (dextran sulfate sodium-induced colitis), IFNL appears to diminish intestinal inflammation, as $\mathrm{In}_{\mathrm{rl}} \mathrm{1}^{-/-}$animals developed greater overall disease scores $(21,75)$, had decreased colon length (76), and had increased expression of oxidative stress genes within the colon (21) when compared with WT animals. Together these data suggest IFNL controls IEC proliferation and suppresses pro-inflammatory immune cells during inflammatory bowel disease. Consistent with this, administration of IFNL to mice with graft-versus-host disease (GVHD) induced by bone marrow transplantation exhibited improved survival, reduced GVHD severity, and enhanced epithelial proliferation and ISC-derived organoid growth after BMT (77).

Genetic variations of IFNL genes have been strongly correlated with outcomes following viral hepatitis. In humans a single nucleotide polymorphism (SNP) of IFNL3 was correlated with viral clearance following hepatitis $C$ treatment with pegylated interferon alpha and ribovarin $(78,79)$. A SNP of IFNL4 also correlated with hepatic inflammation and fibrosis from both viral and non alcoholic fatty liver disease (80).

\section{Central Nervous System Autoimmunity}

Recent work has demonstrated a role for IFNL in the pathogenesis of central nervous system (CNS) autoimmune disease [preprint, (81)]. In the experimental autoimmune encephalomyelitis (EAE) model of multiple sclerosis (MS), Ifnlr $1^{-/-}$animals demonstrated improved clinical disease course and decreased spinal cord axonal injury compared with WT animals. These phenotypes correlated with decreased numbers of Th1 cells, reduced production of proinflammatory cytokines such as IFN $\gamma$ and GMCSF, and diminished activation of antigen presenting cells within the CNS of Ifnlr $1^{-/-}$animals compared to WT animals. Therapeutic targeting of the IFNL 
receptor via antibody mediated neutralization also recapitulated the EAE recovery phenotype observed in $I f n l r 1^{-/-}$animals. Notably, analysis of post-mortem CNS tissue showed increased expression of IFNL and its receptor in lesions of MS patients compared to normal appearing white matter from non MS controls [preprint, (81)].

\section{CONCLUDING REMARKS}

Type III interferon-mediated responses during infections were previously believed to be fairly limited, depending largely on cellspecific expression of the IFNLR, which occurs largely by cells at endothelial and epithelial barriers. However, given the ubiquitous expression of this receptor on both innate and adaptive immune cells, IFNL has increasingly been shown to play a critical role in sculpting overall immune responses during infection and inflammation, promoting Th1 polarization of CD4+ $\mathrm{T}$ cells over Th2. This is especially relevant during certain autoimmune diseases, where IFNL may exert proinflammatory effects. In addition, low levels of expression of IFNL in patients that develop virus-mediated autoreactive diseases at epithelial barriers may underlie tissue damage due to initial lack of virologic control, resulting in autoantigenmediated autoimmunity. Currently, there are no drugs that block IFNL, and, therefore, no clinical trials looking at IFNL as

\section{REFERENCES}

1. Wells AI, Coyne CB. Type III Interferons in Antiviral Defenses at Barrier Surfaces. Trends Immunol (2018) 39:848-58. doi: 10.1016/j.it.2018.08.008

2. Ye L, Schnepf D, Staeheli P. Interferon-Lambda Orchestrates Innate and Adaptive Mucosal Immune Responses. Nat Rev Immunol (2019) 19(10):614-25. doi: 10.1038/ s41577-019-0182-Z

3. Kotenko SV, Gallagher G, Baurin VV, Lewis-Antes A, Shen M, Shah NK, et al. IFN-Lambdas Mediate Antiviral Protection Through a Distinct Class II Cytokine Receptor Complex. Nat Immunol (2003) 4:69-77. doi: 10.1038/ni875

4. Sheppard P, Kindsvogel W, Xu W, Henderson K, Schlutsmeyer S, Whitmore TE, et al. IL-28, IL-29 and Their Class II Cytokine Receptor IL-28r. Nat Immunol (2003) 4:63-8. doi: 10.1038/ni873

5. Prokunina-Olsson L, Muchmore B, Tang W, Pfeiffer R, Park M, Dickensheets $\mathrm{H}$, et al. A Variant Upstream of IFNL3 (IL28B) Creating a New Interferon Gene IFNL4 Is Associated With Impaired Clearance of Hepatitis C Virus. Nat Genet (2013) 45:164-71. doi: 10.1038/ng.2521

6. Lazear HM, Nice TJ, Diamond MS. Interferon-Lambda: Immune Functions at Barrier Surfaces and Beyond. Immunity (2015) 43:15-28. doi: 10.1016/ j.immuni.2015.07.001

7. Syedbasha M, Egli A. Interferon Lambda: Modulating Immunity in Infectious Diseases. Front Immunol (2017) 8:119. doi: 10.3389/fimmu.2017.00119

8. Zhou Z, Hamming OJ, Ank N, Paludan SR, Nielsen AL, Hartmann R. Type III Interferon (IFN) Induces a Type I IFN-Like Response in a Restricted Subset of Cells Through Signaling Pathways Involving Both the Jak-STAT Pathway and the Mitogen-Activated Protein Kinases. J Virol (2007) 81:7749-58. doi: 10.1128/JVI.02438-06

9. Forero A, Ozarkar S, Li H, Lee CH, Hemann EA, Nadjsombati MS, et al. Differential Activation of the Transcription Factor IRF1 Underlies the Distinct Immune Responses Elicited by Type I and Type III Interferons. Immunity (2019) 51:451-64.e456. doi: 10.1016/j.immuni.2019.07.007

10. Sommereyns C, Paul S, Staeheli P, Michiels T. IFN-Lambda (IFN-Lambda) Is Expressed in a Tissue-Dependent Fashion and Primarily Acts on Epithelial a target to treat autoimmune diseases. However, as previously reviewed (54) JAK1 and JAK2 inhibitors are being tested for treatment of SLE. These inhibitors are not specific for IFNLR signaling, as they also target IFNAR. Future studies will define the feasibility of targeting IFNL to prevent chronic inflammatory diseases.

\section{AUTHOR CONTRIBUTIONS}

SM wrote the first draft, performed data mining, generated the figure, and contributed to editing. RK conceived the topic and outline of the review, recruited SM to participate, supervised the preparation of the drafts and figure, wrote the abstract and conclusions, and performed final edits. All authors contributed to the article and approved the submitted version.

\section{FUNDING}

This work was supported by a grant from the National Multiple Sclerosis Society (RG 1801-29766), and grants from the National Institutes of Health (R56AI147623, R35NS122310, R01NS104471, R01NS116788, and R01NS052632; all to RK, and F31 NS108629-01A1; to SM).

Cells In Vivo. PLoS Pathog (2008) 4:e1000017. doi: 10.1371/journal. ppat.1000017

11. Lazear HM, Daniels BP, Pinto AK, Huang AC, Vick SC, Doyle SE, et al. InterferonLambda Restricts West Nile Virus Neuroinvasion by Tightening the Blood-Brain Barrier. Sci Trans Med (2015) 7:284ra259. doi: 10.1126/scitranslmed.aaa4304

12. Read SA, Wijaya R, Ramezani-Moghadam M, Tay E, Schibeci S, Liddle C, et al. Macrophage Coordination of the Interferon Lambda Immune Response. Front Immunol (2019) 10:2674. doi: 10.3389/fimmu.2019.02674

13. Hou W, Wang X, Ye L, Zhou L, Yang ZQ, Riedel E, et al. Lambda Interferon Inhibits Human Immunodeficiency Virus Type 1 Infection of Macrophages. J Virol (2009) 83:3834-42. doi: 10.1128/JVI.01773-08

14. Liu BS, Janssen HL, Boonstra A. IL-29 and IFNalpha Differ in Their Ability to Modulate IL-12 Production by TLR-Activated Human Macrophages and Exhibit Differential Regulation of the IFNgamma Receptor Expression. Blood (2011) 117:2385-95. doi: 10.1182/blood-2010-07-298976

15. de Groen RA, Boltjes A, Hou J, Liu BS, McPhee F, Friborg J, et al. IFNLambda-Mediated IL-12 Production in Macrophages Induces IFN-Gamma Production in Human NK Cells. Eur J Immunol (2015) 45:250-9. doi: 10.1002/eji.201444903

16. Yin Z, Dai J, Deng J, Sheikh F, Natalia M, Shih T, et al. Type III IFNs are Produced by and Stimulate Human Plasmacytoid Dendritic Cells. J Immunol (2012) 189:2735-45. doi: 10.4049/jimmunol.1102038

17. Koltsida O, Hausding M, Stavropoulos A, Koch S, Tzelepis G, Ubel C, et al. IL28a (IFN-Lambda2) Modulates Lung DC Function to Promote Th1 Immune Skewing and Suppress Allergic Airway Disease. EMBO Mol Med (2011) 3:348-61. doi: 10.1002/emmm.201100142

18. Hemann EA, Green R, Turnbull JB, Langlois RA, Savan R, Gale M. InterferonLambda Modulates Dendritic Cells to Facilitate T Cell Immunity During Infection With Influenza A Virus. Nat Immunol (2019) 20:1035-45. doi: 10.1038/s41590-019-0408-z

19. Mennechet FJ, Uze G. Interferon-Lambda-Treated Dendritic Cells Specifically Induce Proliferation of FOXP3-Expressing Suppressor T Cells. Blood (2006) 107:4417-23. doi: 10.1182/blood-2005-10-4129 
20. Kelly A, Robinson MW, Roche G, Biron CA, O'Farrelly C, Ryan EJ. Immune Cell Profiling of IFN-Lambda Response Shows pDCs Express Highest Level of IFNLambdarl and Are Directly Responsive via the JAK-STAT Pathway. J Interferon Cytokine Res (2016) 36:671-80. doi: 10.1089/jir.2015.0169

21. Broggi A, Tan Y, Granucci F, Zanoni I. IFN- $\lambda$ Suppresses Intestinal Inflammation by non-Translational Regulation of Neutrophil Function. Nat Immunol (2017) 18:1084-93. doi: 10.1038/ni.3821

22. Espinosa V, Dutta O, McElrath C, Du P, Chang YJ, Cicciarelli B, et al. Type III Interferon Is a Critical Regulator of Innate Antifungal Immunity. Sci Immunol (2017) 2. doi: 10.1126/sciimmunol.aan5357

23. Syedbasha M, Bonfiglio F, Linnik J, Stuehler C, Wüthrich D, Egli A. Interferon- $\lambda$ Enhances the Differentiation of Naive B Cells Into Plasmablasts via the Mtorc1 Pathway. Cell Rep (2020) 33:108211. doi: 10.1016/j.celrep.2020.108211

24. Marcello T, Grakoui A, Barba-Spaeth G, Machlin ES, Kotenko SV, MacDonald MR, et al. Interferons Alpha and Lambda Inhibit Hepatitis C Virus Replication With Distinct Signal Transduction and Gene Regulation Kinetics. Gastroenterology (2006) 131:1887-98. doi: 10.1053/j.gastro.2006.09.052

25. Jilg N, Lin W, Hong J, Schaefer EA, Wolski D, Meixong J, et al. Kinetic Differences in the Induction of Interferon Stimulated Genes by Interferon- $\alpha$ and Interleukin 28B Are Altered by Infection With Hepatitis C Virus. Hepatol (Baltimore Md) (2014) 59:1250-61. doi: 10.1002/hep.26653

26. Won J, Gil CH, Jo A, Kim HJ. Inhaled Delivery of Interferon-Lambda Restricts Epithelial-Derived Th2 Inflammation in Allergic Asthma. Cytokine (2019) 119:32-6. doi: 10.1016/j.cyto.2019.02.010

27. Dai J, Megjugorac NJ, Gallagher GE, Yu RY, Gallagher G. IFN-Lambda1 (IL29) Inhibits GATA3 Expression and Suppresses Th2 Responses in Human Naive and Memory T Cells. Blood (2009) 113:5829-38. doi: 10.1182/blood2008-09-179507

28. Jordan WJ, Eskdale J, Srinivas S, Pekarek V, Kelner D, Rodia M, et al. Human Interferon Lambda-1 (IFN-Lambda1/IL-29) Modulates the Th1/Th2 Response. Genes Immun (2007) 8:254-61. doi: 10.1038/sj.gene.6364382

29. Egli A, Santer DM, O'Shea D, Barakat K, Syedbasha M, Vollmer M, et al. IL$28 \mathrm{~B}$ is a Key Regulator of B- and T-Cell Vaccine Responses Against Influenza. PLoS Pathog (2014) 10:e1004556. doi: 10.1371/journal.ppat.1004556

30. Morrow MP, Yan J, Pankhong P, Ferraro B, Lewis MG, Khan AS, et al. Unique Th1/Th2 Phenotypes Induced During Priming and Memory Phases by Use of Interleukin-12 (IL-12) or IL-28B Vaccine Adjuvants in Rhesus Macaques. Clin Vaccine Immunol CVI (2010) 17:1493-9. doi: 10.1128/CVI.00181-10

31. Li Y, Gao Q, Yuan X, Zhou M, Peng X, Liu X, et al. Adenovirus Expressing IFN- $\lambda 1$ (IL-29) Attenuates Allergic Airway Inflammation and Airway Hyperreactivity in Experimental Asthma. Int Immunopharmacol (2014) 21:156-62. doi: 10.1016/j.intimp.2014.04.022

32. Blazek K, Eames HL, Weiss M, Byrne AJ, Perocheau D, Pease JE, et al. IFNLambda Resolves Inflammation via Suppression of Neutrophil Infiltration and IL-1beta Production. J Exp Med (2015) 212:845-53. doi: 10.1084/ jem.20140995

33. Srinivas S, Dai J, Eskdale J, Gallagher GE, Megjugorac NJ, Gallagher G. Interferon-Lambda1 (Interleukin-29) Preferentially Down-Regulates Interleukin-13 Over Other T Helper Type 2 Cytokine Responses In Vitro. Immunology (2008) 125:492-502. doi: 10.1111/j.1365-2567.2008.02862.x

34. Hubert M, Gobbini E, Couillault C, Manh TV, Doffin AC, Berthet J, et al. IFNIII is Selectively Produced by Cdc1 and Predicts Good Clinical Outcome in Breast Cancer. Sci Immunol (2020) 5. doi: 10.1126/sciimmunol.aav3942

35. Misumi I, Whitmire JK. IFN-Lambda Exerts Opposing Effects on T Cell Responses Depending on the Chronicity of the Virus Infection. J Immunol (2014) 192:3596-606. doi: 10.4049/jimmunol.1301705

36. Witte K, Gruetz G, Volk HD, Looman AC, Asadullah K, Sterry W, et al. Despite IFN-Lambda Receptor Expression, Blood Immune Cells, But Not Keratinocytes or Melanocytes, Have an Impaired Response to Type III Interferons: Implications for Therapeutic Applications of These Cytokines. Genes Immun (2009) 10:702-14. doi: 10.1038/gene.2009.72

37. Dolganiuc A, Kodys K, Marshall C, Saha B, Zhang S, Bala S, et al. Type III Interferons, IL-28 and IL-29, are Increased in Chronic HCV Infection and Induce Myeloid Dendritic Cell-Mediated FoxP3+ Regulatory T Cells. PLoS One (2012) 7:e44915. doi: 10.1371/journal.pone.0044915

38. Lauterbach H, Bathke B, Gilles S, Traidl-Hoffmann C, Luber CA, Fejer G, et al. Mouse CD8alpha+ DCs and Human BDCA3+ DCs Are Major Producers of
IFN-Lambda in Response to Poly IC. J Exp Med (2010) 207:2703-17. doi: 10.1084/jem.20092720

39. Zhang S, Kodys K, Li K, Szabo G. Human Type 2 Myeloid Dendritic Cells Produce Interferon $-\lambda$ and Amplify Interferon- $\alpha$ in Response to Hepatitis C Virus Infection. Gastroenterology (2013) 144:414-25.e417. doi: 10.1053/ j.gastro.2012.10.034

40. Dufour JH, Dziejman M, Liu MT, Leung JH, Lane TE, Luster AD. IFNGamma-Inducible Protein 10 (IP-10; CXCL10)-Deficient Mice Reveal a Role for IP-10 in Effector T Cell Generation and Trafficking. J Immunol (2002) 168:3195-204. doi: 10.4049/jimmunol.168.7.3195

41. Cole AM, Ganz T, Liese AM, Burdick MD, Liu L, Strieter RM. Cutting Edge: IFN-Inducible ELR- CXC Chemokines Display Defensin-Like Antimicrobial Activity. J Immunol (2001) 167:623-7. doi: 10.4049/ jimmunol.167.2.623

42. Pekarek V, Srinivas S, Eskdale J, Gallagher G. Interferon Lambda-1 (IFNLambda1/IL-29) Induces ELR(-) CXC Chemokine mRNA in Human Peripheral Blood Mononuclear Cells, in an IFN-Gamma-Independent Manner. Genes Immun (2007) 8:177-80. doi: 10.1038/sj.gene.6364372

43. Vasquez RE, Xin L, Soong L. Effects of CXCL10 on Dendritic Cell and CD4+ T-Cell Functions During Leishmania Amazonensis Infection. Infection Immun (2008) 76:161-9. doi: 10.1128/IAI.00825-07

44. Koper OM, Kamińska J, Sawicki K, Kemona H. CXCL9, CXCL10, CXCL11, and Their Receptor (CXCR3) in Neuroinflammation and Neurodegeneration. Adv Clin Exp Med (2018) 27:849-56. doi: 10.17219/acem/68846

45. Goel RR, Wang X, O'Neil LJ, Nakabo S, Hasneen K, Gupta S, et al. Interferon Lambda Promotes Immune Dysregulation and Tissue Inflammation in TLR7Induced Lupus. Proc Natl Acad Sci USA (2020) 117:5409-19. doi: 10.1073/ pnas. 1916897117

46. Liu MQ, Zhou DJ, Wang X, Zhou W, Ye L, Li JL, et al. IFN-Lambda3 Inhibits HIV Infection of Macrophages Through the JAK-STAT Pathway. PLoS One (2012) 7:e35902. doi: 10.1371/journal.pone.0035902

47. Wang Y, Li T, Chen Y, Wei H, Sun R, Tian Z. Involvement of NK Cells in IL28b-Mediated Immunity Against Influenza Virus Infection. J Immunol (2017) 199:1012-20. doi: 10.4049/jimmunol.1601430

48. de Groen RA, Groothuismink ZM, Liu BS, Boonstra A. IFN- $\lambda$ is Able to Augment TLR-Mediated Activation and Subsequent Function of Primary Human B Cells. J Leukocyte Biol (2015) 98:623-30. doi: 10.1189/jlb.3A0215041RR

49. Novak AJ, Grote DM, Ziesmer SC, Rajkumar V, Doyle SE, Ansell SM. A Role for IFN-Lambdal in Multiple Myeloma B Cell Growth. Leukemia (2008) 22:2240-6. doi: 10.1038/leu.2008.263

50. Tsokos GC. Systemic Lupus Erythematosus. N Engl J Med (2011) 365:211021. doi: 10.1056/NEJMra1100359

51. Parodis I, Stockfelt M, Sjöwall C. B Cell Therapy in Systemic Lupus Erythematosus: From Rationale to Clinical Practice. Front Med (2020) 7:316. doi: 10.3389/fmed.2020.00316

52. Wallace DJ. The Evolution of Drug Discovery in Systemic Lupus Erythematosus. Nat Rev Rheumatol (2015) 11:616-20. doi: 10.1038/nrrheum.2015.86

53. Morand EF, Furie R, Tanaka Y, Bruce IN, Askanase AD, Richez C, et al. Trial of Anifrolumab in Active Systemic Lupus Erythematosus. N Engl J Med (2020) 382:211-21. doi: 10.1056/NEJMoa1912196

54. Goel RR, Kotenko SV, Kaplan MJ. Interferon Lambda in Inflammation and Autoimmune Rheumatic Diseases. Nat Rev Rheumatol (2021) 17:349-62. doi: 10.1038/s41584-021-00606-1

55. Wu Q, Yang Q, Lourenco E, Sun H, Zhang Y. Interferon-Lambda1 Induces Peripheral Blood Mononuclear Cell-Derived Chemokines Secretion in Patients With Systemic Lupus Erythematosus: Its Correlation With Disease Activity. Arthritis Res Ther (2011) 13:R88. doi: 10.1186/ar3363

56. Lin SC, Kuo CC, Tsao JT, Lin LJ. Profiling the Expression of Interleukin (IL)28 and IL-28 Receptor $\alpha$ in Systemic Lupus Erythematosus Patients. Eur J Clin Invest (2012) 42:61-9. doi: 10.1111/j.1365-2362.2011.02557.x

57. Zickert A, Oke V, Parodis I, Svenungsson E, Sundström Y, Gunnarsson I. Interferon (IFN)- $\lambda$ is a Potential Mediator in Lupus Nephritis. Lupus Sci Med (2016) 3:e000170. doi: 10.1136/lupus-2016-000170

58. Chen JY, Wang CM, Chen TD, Jan Wu YJ, Lin JC, Lu LY, et al. InterferonLambda3/4 Genetic Variants and Interferon-Lambda3 Serum Levels are Biomarkers of Lupus Nephritis and Disease Activity in Taiwanese. Arthritis Res Ther (2018) 20:193. doi: 10.1186/s13075-018-1683-z 
59. Krasnova TN, Samokhodskaya LM, Ivanitsky LV, Korogodina AD, Borisov EN, Nikiforova NV, et al. Impact of Interleukin-10 and Interleukin-28 Gene Polymorphisms on the Development and Course of Lupus Nephritis. Terapevticheskii Arkhiv (2015) 87:40-4. doi: 10.17116/terarkh201587640-44

60. Zahn S, Rehkämper C, Kümmerer BM, Ferring-Schmidt S, Bieber T, Tüting T, et al. Evidence for a Pathophysiological Role of Keratinocyte-Derived Type III Interferon (Ifn $\lambda)$ in Cutaneous Lupus Erythematosus. J Invest Dermatol (2011) 131:133-40. doi: 10.1038/jid.2010.244

61. Scott DL, Wolfe F, Huizinga TW. Rheumatoid Arthritis. Lancet (London England) (2010) 376:1094-108. doi: 10.1016/S0140-6736(10)60826-4

62. Wang F, Xu L, Feng X, Guo D, Tan W, Zhang M. Interleukin-29 Modulates Proinflammatory Cytokine Production in Synovial Inflammation of Rheumatoid Arthritis. Arthritis Res Ther (2012) 14:R228. doi: 10.1186/ar4067

63. Wu Q, Yang Q, Sun H, Li M, Zhang Y, La Cava A. Serum IFN-Lambdal Is Abnormally Elevated in Rheumatoid Arthritis Patients. Autoimmunity (2013) 46:40-3. doi: 10.3109/08916934.2012.730587

64. Castillo-Martinez D, Juarez M, Patlan M, Paez A, Masso F, Amezcua-Guerra LM, et al. Type-III Interferons and Rheumatoid Arthritis: Correlation Between Interferon Lambda 1 (Interleukin 29) and Antimutated Citrullinated Vimentin Antibody Levels. Autoimmunity (2017) 50:82-5. doi: 10.1080/08916934.2017.1289181

65. Chang QJ, Lv C, Zhao F, Xu TS, Li P. Elevated Serum Levels of Interleukin-29 Are Associated With Disease Activity in Rheumatoid Arthritis Patients With Anti-Cyclic Citrullinated Peptide Antibodies. Tohoku J Exp Med (2017) 241:89-95. doi: 10.1620/tjem.241.89

66. Mustila A, Korpela M, Haapala AM, Kautiainen H, Laasonen L, Möttönen T, et al. Anti-Citrullinated Peptide Antibodies and the Progression of Radiographic Joint Erosions in Patients With Early Rheumatoid Arthritis Treated With FIN-RACo Combination and Single Disease-Modifying Antirheumatic Drug Strategies. Clin Exp Rheumatol (2011) 29:500-5.

67. Xu L, Peng Q, Xuan W, Feng X, Kong X, Zhang M, et al. Interleukin-29 Enhances Synovial Inflammation and Cartilage Degradation in Osteoarthritis. Mediators Inflamm (2016) 2016:9631510. doi: 10.1155/ 2016/9631510

68. Li E, Knight JM, Wu Y, Luong A, Rodriguez A, Kheradmand F, et al. Airway Mycosis in Allergic Airway Disease. Adv Immunol (2019) 142:85-140. doi: 10.1016/bs.ai.2019.05.002

69. Egli A, Santer DM, O'Shea D, Tyrrell DL, Houghton M. The Impact of the Interferon-Lambda Family on the Innate and Adaptive Immune Response to Viral Infections. Emerging Microbes Infect (2014) 3:e51. doi: 10.1038/ emi.2014.51

70. Bullens DM, Decraene A, Dilissen E, Meyts I, De Boeck K, Dupont LJ, et al. Type III IFN-Lambda mRNA Expression in Sputum of Adult and SchoolAged Asthmatics. Clin Exp Allergy J Br Soc Allergy Clin Immunol (2008) 38:1459-67. doi: 10.1111/j.1365-2222.2008.03045.x

71. da Silva J, Hilzendeger C, Moermans C, Schleich F, Henket M, Kebadze T, et al. Raised Interferon- $\beta$, Type 3 Interferon and Interferon-Stimulated Genes Evidence of Innate Immune Activation in Neutrophilic Asthma. Clin Exp Allergy J Br Soc Allergy Clin Immunol (2017) 47:313-23. doi: 10.1111/cea.12809
72. Contoli M, Message SD, Laza-Stanca V, Edwards MR, Wark PA, Bartlett NW, et al. Role of Deficient Type III Interferon-Lambda Production in Asthma Exacerbations. Nat Med (2006) 12:1023-6. doi: 10.1038/nm1462

73. Castillo JR, Peters SP, Busse WW. Asthma Exacerbations: Pathogenesis, Prevention, and Treatment. J Allergy Clin Immunol In Pract (2017) 5:918-27. doi: 10.1016/j.jaip.2017.05.001

74. Chiriac MT, Buchen B, Wandersee A, Hundorfean G, Günther C, Bourjau Y. Activation of Epithelial Signal Transducer and Activator of Transcription 1 by Interleukin 28 Controls Mucosal Healing in Mice With Colitis and Is Increased in Mucosa of Patients With Inflammatory Bowel Disease. Gastroenterology (2017) 153:123-38.e128. doi: 10.1053/j.gastro.2017.03.015

75. Rauch I, Rosebrock F, Hainzl E, Heider S, Majoros A, Wienerroither S, et al. Noncanonical Effects of IRF9 in Intestinal Inflammation: More Than Type I and Type III Interferons. Mol Cell Biol (2015) 35:2332-43. doi: 10.1128/ MCB.01498-14

76. McElrath C, Espinosa V, Lin JD, Peng J, Sridhar R, Dutta O, et al. Critical Role of Interferons in Gastrointestinal Injury Repair. Nat Commun (2021) 12:2624. doi: 10.1038/s41467-021-22928-0

77. Henden AS, Koyama M, Robb RJ, Forero RJ, Kuns RD, Chang K, et al. IFN- $\lambda$ Therapy Prevents Severe Gastrointestinal Graft-Versus-Host Disease. Blood (2021) 138:722-37. doi: 10.1182/blood.2020006375

78. Ge D, Fellay J, Thompson AJ, Simon JS, Shianna KV, Urban TJ, et al. Genetic Variation in IL28B Predicts Hepatitis C Treatment-Induced Viral Clearance. Nature (2009) 461:399-401. doi: 10.1038/nature08309

79. Suppiah V, Moldovan M, Ahlenstiel G, Berg T, Weltman M, Abate ML, et al. IL28B is Associated With Response to Chronic Hepatitis C Interferon-Alpha and Ribavirin Therapy. Nat Genet (2009) 41:1100-4. doi: 10.1038/ng.447

80. Eslam M, Hashem AM, Leung R, Romero-Gomez M, Berg T, Dore GJ, et al. Interferon- $\lambda$ Rs 12979860 Genotype and Liver Fibrosis in Viral and Non-Viral Chronic Liver Disease. Nat Commun (2015) 6:6422. doi: 10.1038/ncomms7422

81. Manivasagam S, Williams JL, Vollmer LL, Bollman B, Bartleson S, Ai S, et al. Targeting Interferon- $\lambda$ Signaling Promotes Recovery From Central Nervous System Autoimmunity. BioRx Preprint. doi: 10.1101/2021.08.17.456642

Conflict of Interest: The authors declare that the research was conducted in the absence of any commercial or financial relationships that could be construed as a potential conflict of interest.

Publisher's Note: All claims expressed in this article are solely those of the authors and do not necessarily represent those of their affiliated organizations, or those of the publisher, the editors and the reviewers. Any product that may be evaluated in this article, or claim that may be made by its manufacturer, is not guaranteed or endorsed by the publisher.

Copyright (c) 2021 Manivasagam and Klein. This is an open-access article distributed under the terms of the Creative Commons Attribution License (CC BY). The use, distribution or reproduction in other forums is permitted, provided the original author(s) and the copyright owner(s) are credited and that the original publication in this journal is cited, in accordance with accepted academic practice. No use, distribution or reproduction is permitted which does not comply with these terms. 